\title{
Evaporative cooling of an atomic beam
}

\author{
E. Mandonnet, A. Minguzzi*, R. Dum, I. Carusotto*, \\ Y. Castin, and J. Dalibard \\ Laboratoire Kastler Brossel, 24 rue Lhomond, 75005 Paris, France \\ * INFM, Scuola Normale Superiore, Piazza dei Cavalieri 7, I-56126 Pisa, Italy
}

\begin{abstract}
We present a theoretical analysis of the evaporative cooling of an atomic beam propagating in a magnetic guide. Cooling is provided by transverse evaporation. The atomic dynamics inside the guide is analyzed by solving the Boltzmann equation with two different approaches: an approximate analytical ansatz and a Monte-Carlo simulation. Within their domain of validity, these two methods are found to be in very good agreement with each other. They allow us to determine how the phase-space density and the flux of the beam vary along its direction of propagation. We find a significant increase for the phase-space density along the guide for realistic experimental parameters. By extrapolation, we estimate the length of the beam needed to reach quantum degeneracy.
\end{abstract}

\section{INTRODUCTION}

Forced evaporative cooling of trapped gases is a very powerful technique to increase the phase-space density of an ensemble of atoms up to quantum degeneracy [1]. Particles with a sufficiently large energy (typically 5 times the thermal energy $k_{B} T$ ) are eliminated. Elastic collisions between the remaining particles restore thermal equilibrium with a lower temperature and an increased phase-space density. The most prominent success of evaporative cooling is the achievement of Bose-Einstein condensation of alkali and hydrogen atomic gases 2 [5].

In this paper we present another possible application of evaporative cooling. We consider atoms moving freely along the $z$-axis and transversely confined by a magnetic field gradient, which provides a harmonic potential in the $x-y$ plane (see fig. 1). The atoms are injected in the plane $z=0$ with given flux, longitudinal and transverse velocity and spatial distributions. We suppose that a radio-frequency field is applied so that only atoms inside a tube-shaped region remain trapped, those outside are evaporated. The bias magnetic field along the $z$-direction is adjusted so that the cross-section of the tube decreases as $z$ increases. As the atoms move forward in the magnetic guide, the ones with a large transverse energy are evaporated. We then rely on elastic collisions between the remaining atoms to decrease the longitudinal velocity width and to increase the phase-space density of the beam.

We investigate theoretically the efficiency of this evaporative cooling scheme by solving the Boltzmann equation with two different approaches: one is based on a truncated distribution ansatz for the phase-space distribution function, while the other one is a direct Monte-Carlo simulation of the atomic dynamics inside the guide. For a suitable geometry of the evaporation scheme, the results of the analytical method fit quite well those of the Monte-Carlo simulation. They show that, with reasonable experimental parameters, the cooling process can lead to a spectacular increase of the phase-space density of the atomic beam. This might be considered as a possible realization of a continuous atom laser [6.7]. Such a coherent source of atoms would have fascinating applications in atom interferometry and holography, metrology and atomic clocks, and nonlinear atom optics [8].

The paper is organized as follows: in section [I we describe the magnetic guide (Sec.II A), we give some typical parameters of the atomic beam source (Sec.IIB), we introduce the Boltzmann equation (Sec.II G), and we detail the evaporation scheme (Sec.IID) ; in section III we use a truncated Boltzmann distribution to obtain an approximate analytical solution of the Boltzmann equation for the case of a $1 \mathrm{D}$ evaporation scheme ; in section [IV we explain the Monte-Carlo simulation and we analyze the results of the two methods in section $\mathrm{V}$. The paper is concluded in section VI with a brief discussion of the coherence properties of the beam when the cooling is sufficiently efficient to reach quantum degeneracy, that is when the spatial density is of the order of $\lambda^{-3}$, where $\lambda$ is the local thermal wavelength.

\section{MODEL CONSIDERED}




\section{A. The atomic guide}

We assume that the magnetic guide consists of four parallel wires carrying the same current $\pm I$ along the $z$ direction (see fig. 11); each wire is at a distance $a$ from the line $x=y=0$. The resulting magnetic field is $\mathbf{B}=b^{\prime}(x,-y, 0)$, with $b^{\prime}=2 \mu_{0} I /\left(\pi a^{2}\right)$. We superimpose a longitudinal magnetic field $\mathbf{B}_{\mathbf{0}}$ along the $z$ axis so that the modulus of the total magnetic field can be written for $x, y$ sufficiently small:

$$
B(\mathbf{r})=\left(B_{0}^{2}+b^{\prime 2}\left(x^{2}+y^{2}\right)\right)^{1 / 2} \simeq B_{0}+\frac{b^{2}}{2 B_{0}}\left(x^{2}+y^{2}\right)
$$

A magnetic moment $\mu$ which is prepared in the direction opposite to the local $\mathbf{B}$ will therefore experience a trapping harmonic potential in the transverse directions. This potential is necessarily isotropic in the $x-y$ plane because of Maxwell equations for magnetostatics. The oscillation frequency in this plane is given by

$$
\Omega_{\perp}=\left(\mu b^{\prime 2} /\left(m B_{0}\right)\right)^{1 / 2}
$$

where $m$ is the atomic mass.

Typical experimental values are $a=4 \mathrm{~mm}, I=500 \mathrm{~A}$, and $B_{0}=1 \mathrm{mT}$. For rubidium atoms with $\mu$ equal to the Bohr magneton, this leads to an oscillation frequency $\Omega_{\perp} \sim 2 \pi \times 1000 \mathrm{~Hz}$. The quadratic expansion leading to (1) is valid for transverse temperatures up to $500 \mu \mathrm{K}$.

\section{B. Parameters of the atomic beam source}

In our model the atoms - for the envisaged experiment we consider rubidium atoms - enter the magnetic guide in the plane $z=0$ with a Gaussian velocity and space distribution with the same initial temperature $T_{0}$ for transverse and longitudinal degrees of freedom. The longitudinal velocity distribution is centered around a non zero value $\bar{v}_{0}$. The ratio $\bar{v}_{0} / \Delta v_{0}$, where $\Delta v_{0}=\sqrt{k_{B} T / m}$ is the r.m.s. of the Gaussian velocity distribution, should be larger than 1 to ensure that only a very small fraction of atoms is initially moving "upstream", that is with a negative longitudinal velocity. We choose in the following

$$
\bar{v}_{0} / \Delta v_{0}=3
$$

For an initial temperature $T_{0}=400 \mu \mathrm{K}$, this gives $\bar{v}_{0}=60 \mathrm{~cm} / \mathrm{s}$. The r.m.s. of the spatial transverse distribution is $R_{\perp 0}=\Delta v_{0} / \Omega_{\perp}$.

We further assume an initial on-axis density $n_{0}$ at $x=y=0$ of the atomic beam of $8 \times 10^{11} \mathrm{~cm}^{-3}$ which corresponds to a flux $\Phi_{0}=2 \pi R_{\perp 0}^{2} n_{0} \bar{v}_{0} \simeq 3 \times 10^{9} \mathrm{~s}^{-1}[9]$. The initial stage of the evaporative cooling within the guide can be described by classical dynamics as $n_{0} \lambda_{0}^{3} \approx 7 \times 10^{-7} \ll 1$, where $\lambda_{0}=h / \sqrt{2 \pi m k_{B} T_{0}}$ is the initial thermal wavelength of the gas.

\section{Collisional dynamics inside the guide}

The collisional dynamics inside the guide can be theoretically described by the Boltzmann equation, which gives the time evolution of the atomic phase-space density $f(\mathbf{r}, \mathbf{p}, t)$ :

$$
\frac{\partial f}{\partial t}+\frac{\mathbf{p}}{m} \cdot \nabla_{\mathbf{r}} f-\nabla_{\mathbf{r}} U \cdot \nabla_{\mathbf{p}} f=I_{\text {coll }}[f]
$$

where $U(x, y, z)=U_{x}(x)+U_{y}(y)$ with $U_{x}(x)=\frac{1}{2} m \Omega_{\perp}^{2} x^{2}$ and $U_{y}(y)=\frac{1}{2} m \Omega_{\perp}^{2} y^{2}$. The distribution $f$ is normalized so that its integral over momentum gives the spatial density. Considering only elastic and isotropic collisions between guided atoms, we write the collisional integral as:

$$
\begin{aligned}
I_{\mathrm{coll}}[f]= & \frac{\sigma}{\pi m^{2}} \int d^{3} p_{2} \int d^{3} p_{3} \int d^{3} p_{4}\left(f\left(\mathbf{r}, \mathbf{p}_{3}\right) f\left(\mathbf{r}, \mathbf{p}_{4}\right)-f(\mathbf{r}, \mathbf{p}) f\left(\mathbf{r}, \mathbf{p}_{2}\right)\right) \\
& \delta\left(\mathbf{p}+\mathbf{p}_{2}-\mathbf{p}_{3}-\mathbf{p}_{4}\right) \delta\left(\frac{p^{2}}{2 m}+\frac{p_{2}^{2}}{2 m}-\frac{p_{3}^{2}}{2 m}-\frac{p_{4}^{2}}{2 m}\right) .
\end{aligned}
$$


Here we assumed that the cross-section $\sigma$ is independent of atomic momentum, a valid assumption for alkali atoms (in the absence of a zero energy resonance) if the temperature is low enough so that collisions essentially occur in the $s$-wave regime. For instance for polarized rubidium atoms, the region of $s$-wave scattering extends up to $400 \mu \mathrm{K}$, corresponding to a r.m.s. velocity $\Delta v_{0}=20 \mathrm{~cm} / \mathrm{s}$. Above this value, $d$-wave scattering is not negligible, and it may significantly modify the results of this paper. We recall that $p$-wave scattering (more generally any odd wave scattering) is forbidden at any energy for polarized bosons.

The collision rate of the atomic beam source $\gamma_{\text {coll }}$ is related to the on-axis density $n_{0}$ by $\gamma_{\text {coll }}=$ $(2 / \sqrt{\pi}) n_{0} \sigma \Delta v_{0}$. For the $s$-wave collisional cross-section of rubidium $\left(\sigma=7.6 \times 10^{-16} \mathrm{~m}^{2}\right)$ we get:

$$
\gamma_{\mathrm{coll}} / \Omega_{\perp}=0.02
$$

which means that an atom performs on average several transverse oscillations between two collisions, that is we are in the collisionless regime for the transverse degrees of freedom.

\section{Evaporation scheme}

Evaporation along the beam eliminates atoms outside a section in the transverse $x y$ plane. In order to optimize the efficiency of this evaporation, we assume that the size of this section varies with $z$ in a controlled way (forced evaporation). In practice this can be achieved using a radio-frequency field at a fixed frequency

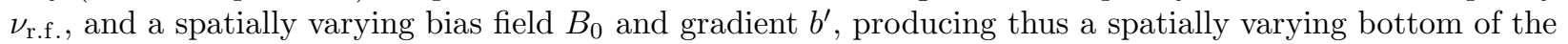
magnetic well while keeping a constant $\Omega_{\perp} \propto b^{\prime} / \sqrt{B_{0}}[10]$. The stability constraints concern mostly the bias field $B_{0}$ as it controls directly the effective local trapping potential depth $\Delta U_{0}=\left(h \nu_{\text {r.f. }} / g\right)-\mu B_{0}$ where $g$ is the Landé factor of the atomic level. As we shall see in section $\S \overrightarrow{\mathrm{VA}}$ a typical value for $\Delta U_{0}$ at the point where quantum degeneracy is reached is $\sim 1 \mu \mathrm{K}$. This value is comparable to the potential depth typically used in Bose-Einstein condensation experiments, so that we do not anticipate any particular difficulty with the control of $B_{0}$. Another possibility to achieve evaporation is to place an absorbing material at a controlled distance $\Lambda_{\text {evap }}(z)$ from the center of the guide.

Evaporation is taken into account in the model by putting $f(\mathbf{r}, \mathbf{p}, t)=0$ if the phase-space cell $\{\mathbf{r}, \mathbf{p}\}$ is outside the domain where atoms are trapped. For the case of a radio-frequency evaporation, which is cylindrically symmetric as long as gravity is negligible, the evaporation criterion is:

$$
x^{2}+y^{2}>\Lambda_{\text {evap }}^{2}(z)
$$

where $\Lambda_{\text {evap }}(z)$ is determined by the radio-frequency field: $g \mu\left(B_{0}^{2}+b^{2} \Lambda_{\text {evap }}^{2}\right)^{1 / 2}=h \nu_{\mathrm{rf}}$. This corresponds to a $2 \mathrm{D}$ evaporation scheme. We also will consider in this paper a $1 \mathrm{D}$ scheme with the criterion

$$
x^{2}>\Lambda_{\text {evap }}^{2}(z)
$$

As we will see below, this 1D scheme allows an approximate analytical treatment. We also define the cut-off energy:

$$
\epsilon_{c}(z)=\frac{1}{2} m \Omega_{\perp}^{2} \Lambda_{\text {evap }}^{2}(z)
$$

\section{1D HYDRODYNAMIC APPROACH}

\section{A. The truncated Boltzmann distribution ansatz}

In this section we restrict the discussion to the 1D scheme of Eq. (8). We now assume that the flow of the gas is in the hydrodynamic regime along the longitudinal direction, that is the macroscopic quantities - such as density or mean velocity - vary slowly with $z$ on a scale given by the mean free path $d(z)=\Delta v(z) / \gamma_{\text {coll }}(z)$. The assumption of a hydrodynamic regime implies a local thermodynamic equilibrium characterized by a local temperature $T(z)$ at abscissa $z$.

We also assume that the mean free path is much larger than the transverse extension $R_{\perp}(z)$ of the beam, or equivalently $\gamma_{\text {coll }}(z) \ll \Omega_{\perp}$. As a consequence, in the $1 \mathrm{D}$ evaporation scheme, if an atom emerges from a 
collision with a kinetic+potential energy along the $x$-axis higher than the cut-off $\epsilon_{c}(z)$, it will fulfil after the further oscillation - and before it undergoes another collision - the condition (8) and it will be evaporated. Therefore it is equivalent to formulate the evaporation criterion either in terms of the coordinate $x$ or in terms of $\epsilon_{x}\left(x, p_{x}\right)=U_{x}(x)+p_{x}^{2} /(2 m)$. Consequently we replace Eq.(8) by

$$
\frac{1}{2} m \Omega_{\perp}^{2} x^{2}+\frac{p_{x}^{2}}{2 m}>\epsilon_{c}(z) .
$$

The assumption of a local thermodynamic equilibrium of the gas at each abscissa $z$ around a mean velocity $\bar{v}(z)=\bar{p}(z) / m$ suggests the following ansatz for the classical phase-space distribution [11]:

$$
f(\mathbf{r}, \mathbf{p})=f_{0}(z) e^{-\beta(z)\left(\epsilon_{x}+\epsilon_{y}\right)} e^{-\beta(z)\left(p_{z}-\bar{p}(z)\right)^{2} / 2 m} Y\left(\epsilon_{c}(z)-\epsilon_{x}\left(x, p_{x}\right)\right) .
$$

Here $\beta(z)=1 /\left(k_{B} T(z)\right)$ and $Y$ is the Heaviside step function. The truncation takes into account the evaporation: $f=0$ for an atom whose energy in the transverse direction $x$ exceeds the local depth $\epsilon_{c}(z)$. The on-axis phase-space density $f_{0}(z)$ is calculated by the normalization condition $\int d x d y \int d^{3} p f=\rho_{\text {lin }}(z)$; here $\rho_{\text {lin }}(z)$ is the linear density of the gas, that is the number of particles per unit length in the guide. A straightforward calculation leads to

$$
f_{0}(z)=\frac{1}{1-e^{-\eta(z)}} \frac{1}{(2 \pi)^{5 / 2}} \frac{\rho_{\operatorname{lin}}(z)}{(m \Delta v(z))^{3} R_{\perp}^{2}(z)}
$$

where we have introduced the thermal velocity $\Delta v(z)=\sqrt{k_{B} T(z) / m}$, the thermal transverse size of the beam $R_{\perp}(z)=\sqrt{k_{B} T(z) / m \Omega_{\perp}^{2}}$, and the quantity

$$
\eta(z)=\epsilon_{c}(z) / k_{B} T(z)
$$

which is a crucial parameter to control the efficiency of evaporation.

This reformulation of the evaporation criterion in the energy domain is difficult to extend to the $2 \mathrm{D}$ scheme. It would require the further assumption of ergodicity of the transverse motion, which is not correct for the present axi-symmetric potential since the knowledge of the total transverse energy $\epsilon_{\perp}=\epsilon_{x}+\epsilon_{y}$ is not a sufficient criterion to determine whether an atomic trajectory will be evaporated. For instance atoms moving along transverse linear trajectories are evaporated when $\epsilon_{\perp}>\epsilon_{c}(z)$, while atoms with transverse circular trajectories remain trapped as long as $\epsilon_{\perp}$ is below $2 \epsilon_{c}(z)$. This $2 \mathrm{D}$ case will be treated using a Monte-Carlo technique in section VB [12].

\section{B. Hydrodynamic equations}

We now derive a closed set of partial differential equations for the three macroscopic quantities $T(z, t)$, $\rho_{\text {lin }}(z, t)$, and $\bar{p}(z, t)$. We note that $f(\mathbf{r}, \mathbf{p})$ in the transverse direction depends only on $\epsilon_{x}$ and $\epsilon_{y}$, quantities preserved by the Hamiltonian evolution. Therefore Eq.(㺼) reduces to

$$
\frac{\partial f}{\partial t}+\frac{p_{z}}{m} \frac{\partial f}{\partial z}=I_{\text {coll }}[f]
$$

We now proceed in a way analogous to the derivation of the standard hydrodynamic equations from the Boltzmann equation: multiplying Eq.(14) by $1, p_{z}$, and $\epsilon_{x}+\epsilon_{y}+p_{z}^{2} / 2 m$, respectively, and integrating over the five variables $x, y, p_{x}, p_{y}, p_{z}$ gives:

$$
\begin{aligned}
\frac{\partial}{\partial t} \rho_{\operatorname{lin}}+\frac{\partial}{\partial z}\left[\frac{\bar{p}}{m} \rho_{\operatorname{lin}}\right] & =-\Gamma \rho_{\text {lin }} \\
\frac{\partial}{\partial t}\left[\bar{p} \rho_{\operatorname{lin}}\right]+\frac{\partial}{\partial z}\left[\left(k_{B} T+\frac{\bar{p}^{2}}{m}\right) \rho_{\operatorname{lin}}\right] & =-\Gamma \bar{p} \rho_{\operatorname{lin}} \\
\frac{\partial}{\partial t}\left[\left(\bar{\epsilon}_{x}+\frac{3}{2} k_{B} T+\frac{\bar{p}^{2}}{2 m}\right) \rho_{\operatorname{lin}}\right]+\frac{\partial}{\partial z}\left[\left(\bar{\epsilon}_{x}+\frac{5}{2} k_{B} T+\frac{\bar{p}^{2}}{2 m}\right) \frac{\bar{p}}{m} \rho_{\operatorname{lin}}\right] & =-\left(\Gamma \frac{\bar{p}^{2}}{2 m}+\Gamma_{\epsilon} k_{B} T\right) \rho_{\operatorname{lin}}
\end{aligned}
$$

where 


$$
\bar{\epsilon}_{x}=\frac{1}{\rho_{\mathrm{lin}}} \int d x d y \int d^{3} p \epsilon_{x} f(\mathbf{r}, \mathbf{p})=k_{B} T\left(1-\frac{\eta}{e^{\eta}-1}\right) .
$$

Equations (15, 16, 17) are the equations of conservation for the number, the momentum, and the energy of the particles. On the right-hand side we have source terms due to evaporation: $\Gamma$ is the evaporation rate at abscissa $z$, and $\Gamma_{\epsilon} k_{B} T$ is the rate for the decrease of energy in the local reference frame moving at velocity $\bar{v}(z)$.

The 1D evaporation model [13] allows to derive explicit expressions for $\Gamma$ and $\Gamma_{\epsilon}$. We obtain for the loss rate of particles:

$$
\Gamma(z)=\sigma \rho_{\operatorname{lin}}(z) \frac{\Delta v(z)}{R_{\perp}^{2}(z)} e^{-\eta(z)} S(\eta(z))
$$

The analytical expression of the positive dimensionless coefficient $S(\eta)$ is given in the appendix. As shown in fig. 2, it is a nearly constant quantity, of the order of 0.075 , when $\eta$ varies between 2 and 10. Eq.(19) shows that the decay rate $\Gamma$ of the linear density, given in eq.(15), is proportional to the collisional cross section $\sigma$ and to the local atomic density, as expected for binary collisions.

For the loss rate of energy, we find:

$$
\Gamma_{\epsilon}(z)=\Gamma(z)\left(\eta(z)+\frac{3}{2}+\tilde{S}(\eta(z))\right) .
$$

The expression of the positive dimensionless coefficient $\tilde{S}(\eta)$ is also derived in the appendix, and plotted in fig 2. It increases from 0.43 to 0.66 when $\eta$ varies from 2 to 10 .

On the left-hand sides of equations (15, 16, 17) we have neglected the terms arising from the $z$-dependence of the cut-off $\epsilon_{c}(z)$. These terms would account for spilling, that is the loss of particles even in absence of collisions due to the lowering of the cut-off $\epsilon_{c}(z)$ along the beam. Neglection of spilling is valid for $\eta \gg 1$, which is well verified for the optimal evaporation with realistic initial parameters; for instance, for the simulations presented in section [V], we have chosen $\eta \simeq 5$ [14]. For consistency we also replace in the following $\bar{\epsilon}_{x}$ and $f_{0}$ by their values for $\eta \rightarrow \infty$ :

$$
\begin{aligned}
\bar{\epsilon}_{x} & =k_{B} T \\
f_{0}(z) & =\frac{1}{(2 \pi)^{5 / 2}} \frac{\rho_{\operatorname{lin}}(z)}{(m \Delta v(z))^{3} R_{\perp}^{2}(z)}
\end{aligned}
$$

We finally obtain from Eqs. (15,16,17) in the limit of large $\eta$ :

$$
\begin{aligned}
\left(\frac{\partial}{\partial t}+\frac{\bar{p}}{m} \frac{\partial}{\partial z}\right) \rho_{\operatorname{lin}}+\rho_{\operatorname{lin}} \frac{\partial}{\partial z}\left[\frac{\bar{p}}{m}\right] & =-\Gamma \rho_{\operatorname{lin}} \\
\left(\frac{\partial}{\partial t}+\frac{\bar{p}}{m} \frac{\partial}{\partial z}\right) \bar{p}+\frac{1}{\rho_{\operatorname{lin}}} \frac{\partial}{\partial z}\left[k_{B} T \rho_{\operatorname{lin}}\right] & =0 \\
\left(\frac{\partial}{\partial t}+\frac{\bar{p}}{m} \frac{\partial}{\partial z}\right) k_{B} T+\frac{2}{5} k_{B} T \frac{\partial}{\partial z}\left[\frac{\bar{p}}{m}\right] & =k_{B} T\left(\Gamma-\frac{2}{5} \Gamma_{\epsilon}\right)
\end{aligned}
$$

\section{Stationary regime: $z$-dependence of phase space density}

We are mainly interested in a stationary regime for which we obtain a set of non-linear equations which can be solved by standard numerical methods:

$$
\begin{aligned}
\frac{\partial}{\partial z}\left(\rho_{\operatorname{lin}}\right) & =-\frac{m}{\bar{p}} \rho_{\operatorname{lin}}\left(\Gamma+\Gamma_{\epsilon} \frac{k_{B} T}{E}\right) \\
\frac{\partial}{\partial z}\left(\frac{\bar{p}}{m}\right) & =\Gamma_{\epsilon} \frac{k_{B} T}{E} \\
\frac{\partial}{\partial z}\left(k_{B} T\right) & =-\frac{m k_{B} T}{\bar{p}}\left(\frac{2}{5} \Gamma_{\epsilon}-\Gamma+\frac{2}{5} \Gamma_{\epsilon} \frac{k_{B} T}{E}\right)
\end{aligned}
$$


where we have put

$$
E \equiv \frac{5}{2} \frac{\bar{p}^{2}}{m}-\frac{7}{2} k_{B} T
$$

From the above we see that if $\bar{p}^{2} / 2 m$ is bigger than $(7 / 10) k_{B} T$ (i.e. $E>0$ ), which is indeed the parameter range studied in this article, the mean velocity increases as a function of $z$. An interpretation of this result will be given in section $\mathrm{VA}$.

A figure of merit for our scheme is the degree of increase of the phase-space density along the beam. As a measure of this increase we take the on-axis phase-space density $f_{0}(z) \propto \rho_{\operatorname{lin}} /\left(k_{B} T\right)^{5 / 2}$ :

$$
\frac{\partial}{\partial z}\left[\ln \frac{\rho_{\operatorname{lin}}}{\left(k_{B} T\right)^{5 / 2}}\right]=(\eta+\tilde{S}(\eta)-2) \frac{m}{\bar{p}} \Gamma .
$$

Since the quantity $\tilde{S}(\eta)$ is positive (see fig. 2), we conclude that the phase-space density increases when $\eta>2$.

\section{MONTE-CARLO SIMULATIONS}

In this section we compare results from the approximate analytical ansatz of the previous section with results of a Monte-Carlo simulation. The fact that the Monte-Carlo simulation requires a long computing time restricts the parameter space which can be explored. In particular, as explicited below, the length of the guide influences the memory requirement of the Monte-Carlo simulation. The total length $L$ of the system, expressed in units of the mean free path $d_{0}=\sqrt{\pi} /\left(2 n_{0} \sigma\right)$ is chosen in the following as

$$
L / d_{0}=2500 \text {. }
$$

For the parameters given in section $\amalg \mathrm{IB}$ this corresponds to a length of 3.7 meters; the average time $T=L / \bar{v}_{0}$ for an atom to travel from the entrance to the exit of the guide is $T=4 \times 10^{4} \Omega_{\perp}^{-1}$ (in absence of collisions and evaporation); this time corresponds to $830 \gamma_{\text {coll }}^{-1}$.

\section{A. Principle of the Monte-Carlo method}

This method has originally been introduced in the context of molecular dynamics [15]. For the case of dilute gases, it relies on the idea that one can separate the description of the collision from that of the motion, allowing a simulation of the dynamics on a time scale shorter than the mean time between two collisions.

In essence the approach consists in solving (4) numerically by evolving macro-atoms, each of which representing $\ell$ real atoms. The macro-atoms evolve in the same potential as the real atoms, and they have the same initial velocity and position distributions. Their collisional cross-section is $\ell \sigma$ and their initial spatial density is $n_{0} / \ell$, so that the collision rate $\gamma_{\text {coll }}$ and the two dimensionless parameters $\bar{v}_{0} / \Delta v_{0}$ and $L / d_{0}$ are not changed. This approach is valid since the Boltzmann equation is invariant under the scaling $\sigma \rightarrow \ell \sigma$, $f \rightarrow f / \ell$. Using the symmetries of the problem we restrict the evolution to the first quarter $(x>0, y>0)$ of the entrance plane $z=0$ reducing the memory requirement by a factor 4 . We evolve the macro-atoms in this first quarter with reflecting walls at the planes $x=0$ and $y=0$.

We inject on average 84 macro-atoms ( 21 in the first quarter) every $\Omega_{\perp}^{-1}$. We take $\ell=5600$ to match the flux of the atomic beam source presented in section II B. In absence of evaporation, $N=21 \times 4 \times 10^{4}=$ $8.4 \times 10^{5}$ macro-atoms are present on average at a given time since, as stated above, it takes on average $4 \times 10^{4} \Omega_{\perp}^{-1}$ for a particle to travel along the guide.

Binary elastic collisions are taken into account using a boxing technique 16 18]. We introduce in position space a lattice with a unit cell volume $\delta V$, chosen such that the average occupation $p_{\text {occ }}$ of any cell is much smaller than 1. Collisions occur only between two macro-atoms occupying the same cell, and the time step $\delta t$ is adjusted in such a way that the probability $p_{\text {coll }}$ of a collisional event during $\delta t$ is also much smaller than 1 . We choose typically $p_{\text {occ }} \sim p_{\text {coll }} \sim 10 \%$.

Evaporation is implemented in the simulation by eliminating the macro-atoms whose coordinates fulfil the chosen condition of evaporation. In particular, we have treated both the case of evaporation with a 
1D position cut (Eq. (8)) in order to compare with the results of the analytical ansatz, and the case of evaporation with a 2D position cut (Eq. (7)).

We let the simulation evolve until a steady-state is reached. The corresponding time is $\sim 2 L / \bar{v}_{0}=$ $8 \times 10^{4} \Omega_{\perp}^{-1}$. This allows to obtain the average energies along each axis and the linear density at a given

location $z$. From these quantities we can predict the decrease in temperature, the loss of particles and in consequence the phase-space density increase.

\section{B. Comparison with the hydrodynamic approach for 1D evaporation}

The results of the Monte-Carlo and of the hydrodynamic approaches for 1D evaporation are plotted in Figs. 3, 1 and 5, giving the variations with $z$ of the mean velocity $\bar{v}(z)$, the flux $\Phi(z)$, and the phase-space density $f_{0}(z)$. This set of data has been obtained by choosing an evaporation barrier $\epsilon_{c}(z)$ approximately 5 times larger than the mean local transverse energy, that is we kept $\eta \simeq 5$; as shown below, this value for $\eta$ leads to a gain of 7 orders of magnitude for the phase space density with a minimal length requirement.

Using the Monte-Carlo simulation, we have found that the three components of the velocities have a nearly Gaussian distribution, with dispersions equal to within a few percent. This is consistent with the hypothesis of local thermodynamic equilibrium at the basis of the hydrodynamic approach.

The results shown in figs. 3, 4 and 5 show an excellent agreement between the hydrodynamic approach and the Monte-Carlo simulation. This allows one to make all optimization and design procedures for the choice of initial parameters and spatial variations of the cut using the approximate hydrodynamic treatment while keeping the Monte-Carlo simulation - which requires several days of computation on a work station for final checks.

\section{DISCUSSION OF THE RESULTS}

\section{A. 1D evaporation}

Using the equations (26 28) in the regime of evaporation with a constant $\eta$, we have first determined the optimal choice for $\eta$. As shown in fig. 6, the shortest distance providing a $10^{7}$ gain in phase space density - required in order to reach quantum degeneracy for the initial conditions considered in $\S 2$ - is obtained for $\eta \sim 5$. If smaller phase space gain are needed, smaller values of $\eta$ would be more appropriate since they would lead to shorter cooling lengths.

We now consider fig. 3, obtained for $\eta=5$, which shows a slight increase of the mean velocity $\bar{v}(z)$, as the atoms progress within the guide. This increase can be understood from kinematic arguments: the cooling along $z$ can be seen very crudely as a process in which a fast and a slow particle collide, one of them being eliminated. Now, at a given location, the fast particles are renewed with a larger rate than the slow ones, because it takes less time for them to go from the entrance plane to the considered location. Therefore, the mean velocity in a location $z>0$, where particles have already undergone in average several collisions, is larger than in $z=0$. This acceleration effect becomes negligible as soon as $k_{B} T(z) \ll \bar{p}^{2} /(2 m)$ since the beam is then quasi mono-kinetic.

The figs. 1,5 show the flux and the phase-space density as a function of $z$ for $\eta=5$. A very significant increase of phase-space density by a factor 500 proves the efficiency of the evaporative cooling. This increase is accompanied with a reduction of the flux by a factor 5.5. The phase-space density and the flux both vary quasi-exponentially with the position $z$.

To get a better understanding of the variations with $z$ of these quantities, we now consider Eqs. (26-28) in the limiting case where $\bar{v}$ is constant and $k_{B} T \ll E$. Assuming also a constant $\eta$, the solutions of these equations are:

$$
\rho_{\operatorname{lin}}(z)=\rho_{\operatorname{lin}}(0)\left(1-\frac{z}{z_{c}}\right)^{\alpha_{c}} \quad T(z)=T(0)\left(1-\frac{z}{z_{c}}\right)^{\beta_{c}}
$$

with

$$
z_{c}=d_{0} \frac{\bar{v}}{\Delta v_{0}} \frac{5 e^{\eta}}{\pi^{3 / 2} S(\eta)(\eta+\tilde{S}(\eta)-6)} \quad \alpha_{c}=\frac{5}{\eta+\tilde{S}(\eta)-6} \quad \beta_{c}=\frac{2(\eta+\tilde{S}(\eta)-1)}{\eta+\tilde{S}(\eta)-6}
$$


For the parameters of figs.3,4 4 and 5, where $\eta=5$, we find $\alpha_{c} \simeq-12, \beta_{c} \simeq-21.9$ and $z_{c} \simeq$ $-4300 d_{0} \bar{v} / \Delta v_{0} \simeq-13000 d_{0}$, so that the relevant range of lengths $z$ are much smaller than $\left|z_{c}\right|$. Consequently we can approximate the previous results with:

$$
\rho_{\operatorname{lin}}(z) \simeq \rho_{\operatorname{lin}}(0) \exp \left(-\alpha_{c} z / z_{c}\right) \quad T(z) \simeq T(0) \exp \left(-\beta_{c} z / z_{c}\right)
$$

This leads to $\operatorname{exponential~variations~} \exp \left(z / z_{\text {p.s. }}\right)$ and $\exp \left(-z / z_{\text {flux }}\right)$ of the phase space density and the flux, with:

$$
z_{\text {p.s. }}=d_{0} \frac{\bar{v}}{\Delta v_{0}} \frac{e^{\eta}}{\pi^{3 / 2}(\eta+\tilde{S}(\eta)-2) S(\eta)} \quad z_{\text {flux }}=d_{0} \frac{\bar{v}}{\Delta v_{0}} \frac{e^{\eta}}{\pi^{3 / 2} S(\eta)}
$$

This explains why the results in figs. 4 , exhibit a quasi-exponential variation with $z$. For $\eta=5$, we get $z_{\text {p.s. }} \simeq 100 d_{0} \bar{v} / \Delta v_{0}$ and $z_{\text {flux }} \simeq 360 d_{0} \bar{v} / \Delta v_{0}$. The factor 3.6 between these two lengths suggests that a phase space gain of 7 orders of magnitude can be achieved with a flux reduction by less than 2 orders of magnitude. This is confirmed by the numerical solution of the hydrodynamical equations Eq.(26-28): the required length is $7600 d_{0}$, on the order of 11 meters for the parameters of section II; it is accompanied by a decrease of temperature by a factor 4000 and of flux by a factor 90 .

It is worth noting that the collision rate $\gamma_{\text {coll }}(z)$ does not vary much for $z \ll\left|z_{c}\right|$. Indeed, we have:

$$
\gamma_{\text {coll }}(z)=\gamma_{\text {coll }}(0)\left(1-\frac{z}{z_{c}}\right)^{\alpha_{c}-\frac{\beta_{c}}{2}}=\gamma_{\text {coll }}(0) \frac{z_{c}}{z_{c}-z}
$$

The sign of $z_{c}$ is the same as the sign of $\eta+\tilde{S}(\eta)-6$, which vanishes for $\eta \simeq 5.4$. Therefore, the collision rate $\gamma_{\text {coll }}$ increases with $z$ if $\eta>5.4$ and decreases if $\eta<5.4$. For the particular value $\eta=5.4$, the collision rate is constant, as well as the loss rates for particles $\Gamma(z)$ and energy $\Gamma_{\epsilon}(z)$; the quantities $\rho_{\operatorname{lin}}(z)$ and $k_{B} T(z)$ then have an exponential variation for any $z$, since $z_{c}, \alpha_{c}, \beta_{c} \rightarrow \infty$.

\section{B. $2 \mathrm{D}$ versus $1 \mathrm{D}$ evaporation scheme}

The experimental setup based on radio-frequency evaporation and discussed in section II], corresponds to a $2 \mathrm{D}$ evaporation scheme while the analytical treatment of section III is based on a $1 \mathrm{D}$ evaporation. One does not expect the two situations to be equivalent. More precisely, since the $x-y$ degrees of freedom are not mixed by the collisionless motion in the axi-symmetric potential, the $1 \mathrm{D}$ evaporation is expected to be less efficient than a 2D evaporation scheme: a particle may emerge from an elastic collision with a large transverse kinetic energy along the $y$-axis, without being evaporated in the 1D evaporative scheme.

We have checked with our Monte-Carlo simulation that 2D evaporation is more efficient indeed than $1 \mathrm{D}$ evaporation. We have first used a crude model of $2 \mathrm{D}$ evaporation, with the truncation of Eq.(11) replaced by $Y\left(\epsilon_{c}(z)-\epsilon_{\mathrm{tot}}+k_{B} T(z) / 2\right)$, where $\epsilon_{\mathrm{tot}}=\epsilon_{\perp}+\left(p_{z}-\bar{p}(z)\right)^{2} / 2 m$ is the total energy in the frame moving at velocity $\bar{p}(z) / m$. This truncation assumes in particular quasi-ergodicity in the $x-y$ plane. Within this model we perform the same optimization as in fig. 6; to achieve a gain in phase space density by 7 orders of magnitude the optimal $\eta$ is now equal to 6 . We have then run the Monte-Carlo simulation for this value of $\eta$. The gain of a factor 500 in phase space density, which was obtained for a length $2500 d_{0}$ for $1 \mathrm{D}$ evaporation (see fig. 5), is reached now for a length $900 d_{0}$; the relative variation of the flux (reduction by a factor $\sim 6$ ) is similar. The variations with $z$ of the phase space density and the flux are quasi-exponential. Note that the predictions of the crude model are in amazingly good agreement with the Monte-Carlo results.

To conclude the length of the guide needed to achieve a specified gain of the phase-space density is a factor of $\sim 3$ smaller for 2D than for 1D evaporation. E. g. the 11 meters of evaporation length necessary in 1D for the experimental conditions of $\S$ III are now reduced to 4 meters.

\section{PERSPECTIVES}

For a typical experimental source of cold atoms, the length needed for a phase-space increase of seven orders of magnitude - which should bring the system close to the degeneracy point - is of the order of a few meters. The possibility of quantum degeneracy in such a system raises interesting questions. As it is well 
known [19], there is no Bose-Einstein condensation in the thermodynamic limit in a $1 \mathrm{D}$ geometry, obtained here 20 in setting $N, L \rightarrow \infty$, while keeping a constant linear density $\rho_{\text {lin }}=N / L$, a constant temperature $T$ and a constant transverse oscillation frequency $\Omega_{\perp}$. Therefore we do not expect a macroscopic occupation of a single quantum state of the longitudinal motion.

In order to get more insight in the output of this system, we assume that the transverse extension of the thermal component of the beam $R_{\perp}=\sqrt{k_{B} T /\left(m \Omega_{\perp}^{2}\right)}$ is much larger than the thermal wavelength $\lambda=$ $h / \sqrt{2 \pi m k_{B} T}$ (that is $k_{B} T \gg \hbar \Omega_{\perp}$ ). In this case, elastic collisions ensure that thermodynamic equilibrium is reached in the frame moving with the mean velocity of the gas. In this frame, we describe the properties of the system using as an approximation the grand canonical Bose-Einstein distribution for an ideal gas. The gas being at temperature $T$, we obtain the maximal linear density that can be put in states corresponding to an excited transverse motion

$$
\rho_{\text {lin }}^{(c)}=\frac{1}{\lambda} \zeta(5 / 2)\left(\frac{k_{B} T}{\hbar \Omega_{\perp}}\right)^{2}
$$

with $\zeta(5 / 2) \simeq 1.34$. When one increases the linear density above this critical value (which corresponds to a spatial density on axis larger than $\left.n^{(c)}=\zeta(3 / 2) \lambda^{-3}\right)$, the transverse degrees of freedom undergo a Bose-Einstein condensation [21], that is atoms start accumulating in states corresponding to the ground transverse motion (see fig. 7). By convention, we set the energy of this ground state to zero. Since the chemical potential $\mu$ then satisfies $|\mu| \ll \hbar \Omega_{\perp} \ll k_{B} T$, the longitudinal momentum distribution for these atoms can be approximated as:

$$
n(p)=\frac{1}{h} \frac{1}{\exp \left[\left(p^{2} /(2 m)-\mu\right) / k_{B} T\right]-1} \simeq \frac{h}{\pi \lambda^{2}} \frac{1}{p^{2}+p_{c}^{2}}
$$

that is a Lorentzian distribution of half width $p_{c}=[-2 m \mu]^{1 / 2}$. Such a distribution leads to a spatial correlation length of the gas along $z$ given by $\xi_{c}=\hbar / p_{c}$. By integrating $n(p)$ over $p$, we can relate $\mu$ and therefore $\xi_{c}$ to the linear density of atoms in the transverse ground state $\rho_{\text {lin }}-\rho_{\operatorname{lin}}^{(c)}$ :

$$
\xi_{c}=\frac{\lambda^{2}}{2 \pi}\left(\rho_{\text {lin }}-\rho_{\text {lin }}^{(c)}\right)=\frac{1}{2} \zeta(3 / 2) \frac{\hbar}{m \Omega_{\perp} \lambda} \frac{n-n^{(c)}}{n^{(c)}}
$$

where $n$ is the $3 \mathrm{D}$ on-axis density. This correlation length can be much larger than the thermal wavelength. Thus, for $\rho_{\text {lin }}>\rho_{\text {lin }}^{(c)}$, the output of the system can be viewed as propagating independent trains of matter waves, each of which having a length of the order of $\xi_{c}$ and containing on average $\left[\lambda\left(\rho_{\text {lin }}-\rho_{\text {lin }}^{(c)}\right)\right]^{2} /(2 \pi)$ atoms.

To conclude, we have presented in this paper the principles of the evaporative cooling of an atomic beam. In the classical regime, where the mean interparticle distance is much larger than the thermal wavelength $\lambda$, we have evaluated the characteristic lengths for the flux and the phase space density variations. We have also outlined briefly the coherence properties of the beam once quantum degeneracy is reached. A more detailed characterization of these coherence properties, including the effect of interactions between particles, will be the subject of a future work.

\section{ACKNOWLEDGMENTS}

We thank F. Chevy, C. Cohen-Tannoudji, D. Guéry-Odelin, K. Madison, C. Salomon, and G. Shlyapnikov for several helpful discussions. E.M. also acknowledges useful discussions with A. Aspect and P. Bouyer. Laboratoire Kastler Brossel is a unité de recherche du CNRS, associated with Ecole Normale Supérieure and Université Pierre et Marie Curie. A.M. and I. C. are with the Istituto Nazionale per la Fisica della Materia. This work was partially supported by CNRS, Collège de France, DRET, DRED and EC (TMR network ERB FMRX-CT96-0002). 


\section{APPENDIX}

In this appendix we derive explicit expressions for the loss rates for particles $\Gamma$ and for energy $\Gamma_{\epsilon}$ in Eqs. $(15,16,17)$. Integrating (4) over $x, y$, and $\mathbf{p}$ to derive (15), we obtain:

$$
\begin{aligned}
\Gamma(z) \rho_{\operatorname{lin}}(z)= & \frac{\sigma}{\pi m^{2}} \int d x d y \int d^{3} p \int d^{3} p_{2} \int d^{3} p_{3} \int d^{3} p_{4}\left(f(\mathbf{r}, \mathbf{p}) f\left(\mathbf{r}, \mathbf{p}_{2}\right)-f\left(\mathbf{r}, \mathbf{p}_{3}\right) f\left(\mathbf{r}, \mathbf{p}_{4}\right)\right) \\
& \delta\left(\mathbf{p}+\mathbf{p}_{2}-\mathbf{p}_{3}-\mathbf{p}_{4}\right) \delta\left(\frac{p^{2}}{2 m}+\frac{p_{2}^{2}}{2 m}-\frac{p_{3}^{2}}{2 m}-\frac{p_{4}^{2}}{2 m}\right)
\end{aligned}
$$

In this integral, the phase space cell $(\mathbf{r}, \mathbf{p})$ is the variable of integration of the left hand side of (4) and it is always in the trappable domain defined by:

$$
\frac{p_{x}^{2}}{2 m}+\frac{1}{2} m \Omega_{\perp}^{2} x^{2}<\epsilon_{c}(z)
$$

For the first part of the integral, representing the collision $\mathbf{p}+\mathbf{p}_{2} \rightarrow \mathbf{p}_{3}+\mathbf{p}_{4}$ and involving $f(\mathbf{r}, \mathbf{p}) f(\mathbf{r}, \mathbf{p} 2)$, the cell $\left(\mathbf{r}, \mathbf{p}_{2}\right)$ is also in the trappable domain, while the two cells $\left(\mathbf{r}, \mathbf{p}_{3}\right)$ and $\left(\mathbf{r}, \mathbf{p}_{4}\right)$ may be either in or out of the trappable domain. For the second part of the integral, representing the collision $\mathbf{p}_{3}+\mathbf{p}_{\mathbf{4}} \rightarrow \mathbf{p}+\mathbf{p}_{\mathbf{2}}$ and involving $f\left(\mathbf{r}, \mathbf{p}_{3}\right) f\left(\mathbf{r}, \mathbf{p}_{4}\right)$, the two cells $\left(\mathbf{r}, \mathbf{p}_{3}\right)$ and $\left(\mathbf{r}, \mathbf{p}_{4}\right)$ are in the trappable domain, while the cell $\left(\mathbf{r}, \mathbf{p}_{2}\right)$ may be either in or out of the trappable domain.

We now rearrange the second part of this integral by exchanging the role of $\left(\mathbf{p}, \mathbf{p}_{2}\right)$ and $\left(\mathbf{p}_{3}, \mathbf{p}_{4}\right)$. After cancellation of various terms, we are left with:

$$
\begin{aligned}
\Gamma(z) \rho_{\operatorname{lin}}(z)= & \frac{\sigma}{\pi m^{2}} \int d x d y \int d^{3} p_{1} \int d^{3} p_{2} \int d^{3} p_{3} \int d^{3} p_{4} f\left(\mathbf{r}, \mathbf{p}_{\mathbf{1}}\right) f\left(\mathbf{r}, \mathbf{p}_{2}\right) \\
& \delta\left(\mathbf{p}_{1}+\mathbf{p}_{2}-\mathbf{p}_{3}-\mathbf{p}_{4}\right) \delta\left(\frac{p_{1}^{2}}{2 m}+\frac{p_{2}^{2}}{2 m}-\frac{p_{3}^{2}}{2 m}-\frac{p_{4}^{2}}{2 m}\right) .
\end{aligned}
$$

where $\left(\mathbf{r}, \mathbf{p}_{1}\right)$ and $\left(\mathbf{r}, \mathbf{p}_{2}\right)$ are in the trappable domain, $\left(\mathbf{r}, \mathbf{p}_{3}\right)$ is out of the trappable domain, and $\left(\mathbf{r}, \mathbf{p}_{4}\right)$ is either in or out of the trappable domain.

We now put

$$
\mathbf{P}=\frac{\mathbf{p}_{1}+\mathbf{p}_{2}}{2} \quad \mathbf{q}=\frac{\mathbf{p}_{1}-\mathbf{p}_{2}}{2} \quad \mathbf{P}^{\prime}=\frac{\mathbf{p}_{3}+\mathbf{p}_{4}}{2} \quad \mathbf{q}^{\prime}=\frac{\mathbf{p}_{3}-\mathbf{p}_{4}}{2}
$$

The $\delta$-distributions entering into (40) impose $\mathbf{P}=\mathbf{P}^{\prime}$ and $|\mathbf{q}|=\left|\mathbf{q}^{\prime}\right|$. We perform some rearrangements, using as integration variables for the vectors $\mathbf{q}$ and $\mathbf{q}^{\prime}$ the coordinates $q_{x}, q_{x}^{\prime}$ along $x$, the moduli $q, q^{\prime}$ and the azimuthal angles around $x$-axis. We integrate over those angles and we split the integration domain into a part where only particle 3 escapes and a part where both 3 and 4 escape. We then obtain:

$$
\begin{aligned}
\Gamma(z) \rho_{\operatorname{lin}}(z)= & 64 \pi \frac{\sigma}{m} f_{0}^{2}(z) \int_{-x_{c}}^{x_{c}} d x \int_{0}^{Q} d P_{x} \int_{-\infty}^{\infty} d y \int_{-\infty}^{\infty} d P_{y} \int_{-\infty}^{\infty} d P_{z} e^{-2\left(U_{x}+U_{y}\right) / k_{B} T} e^{-P^{2} / m k_{B} T} \\
\times \int_{0}^{Q-P_{x}} d q_{x} & \left\{\int_{Q-P_{x}}^{Q+P_{x}} d q q e^{-q^{2} / m k_{B} T} \int_{Q-P_{x}}^{q} d q_{x}^{\prime}\right. \\
& \left.+\int_{Q+P_{x}}^{\infty} d q q e^{-q^{2} / m k_{B} T}\left[\int_{Q-P_{x}}^{Q+P_{x}} d q_{x}^{\prime}+2 \int_{Q+P_{x}}^{q} d q_{x}^{\prime}\right]\right\}
\end{aligned}
$$

where $P=|\mathbf{P}|$ and $q=|\mathbf{q}|$. The length $x_{c}(z)$ is the positive solution of $m \Omega_{\perp}^{2} x_{c}^{2}(z)=2 \epsilon_{c}(z)$, and $Q(x, z)$ is the local escape momentum given by $Q^{2} / 2 m=\epsilon_{c}(z)-U_{x}(x)$. The expression (42) is the direct transcription of Eq. (26) of [13], to the situation considered in the present paper.

The integrations over $y, P_{y}, P_{z}$ are immediate, as well as for $q_{x}$. After an integration by part over $q$, we get:

$$
\Gamma \rho_{\operatorname{lin}}=16 \pi^{3} \sigma\left(k_{B} T\right)^{9 / 2} m^{3 / 2} \Omega_{\perp}^{-2} f_{0}^{2} \int_{-\sqrt{2 \eta}}^{\sqrt{2 \eta}} d w e^{-w^{2}} \int_{0}^{a} d u(a-u) e^{-u^{2}}(\operatorname{erfc}(a-u)+\operatorname{erfc}(a+u))
$$


where we have put $w=x / R_{\perp}, a=Q / \sqrt{m k T}=\sqrt{2 \eta-w^{2}}, u=P_{x} / \sqrt{m k T}$ and:

$$
\operatorname{erfc}(u)=\frac{2}{\sqrt{\pi}} \int_{u}^{\infty} d v e^{-v^{2}} .
$$

Using finally the relation (12) to express $f_{0}$ in terms of the linear density $\rho_{\text {lin }}$, we arrive to (19) with:

$$
S(\eta)=\frac{1}{2 \pi^{2}} \frac{e^{\eta}}{\left(1-e^{-\eta}\right)^{2}} \int_{-\sqrt{2 \eta}}^{\sqrt{2 \eta}} d w e^{-w^{2}} \int_{0}^{a} d u(a-u) e^{-u^{2}}(\operatorname{erfc}(a-u)+\operatorname{erfc}(a+u)) .
$$

The calculation of $\Gamma_{\epsilon}$ proceeds along the same line. We multiply (4) by $\epsilon_{x}+\epsilon_{y}+p_{z}^{2} / 2 m$ and integrate over $x, y$, and $\mathbf{p}$. Using the same rearrangement as above, we get:

$$
\begin{aligned}
\Gamma_{\epsilon} k_{B} T \rho_{\mathrm{lin}}=64 \pi \frac{\sigma}{m} f_{0}^{2} \int_{-x_{c}}^{x_{c}} d x \int_{0}^{Q} d P_{x} \int_{-\infty}^{\infty} d y \int_{-\infty}^{\infty} d P_{y} \int_{-\infty}^{\infty} d P_{z} e^{-2\left(U_{x}+U_{y}\right) / k_{B} T} e^{-P^{2} / m k_{B} T} \\
\times \int_{0}^{Q-P_{x}} d q_{x}\left\{\int_{Q-P_{x}}^{Q+P_{x}} d q q e^{-q^{2} / m k_{B} T} \int_{Q-P_{x}}^{q} E_{3} d q_{x}^{\prime}\right. \\
\left.+\int_{Q+P_{x}}^{\infty} d q q e^{-q^{2} / m k_{B} T}\left[\int_{Q-P_{x}}^{Q+P_{x}} E_{3} d q_{x}^{\prime}+\int_{Q+P_{x}}^{q}\left(E_{3}+E_{4}\right) d q_{x}^{\prime}\right]\right\}
\end{aligned}
$$

where:

$$
\begin{aligned}
& E_{3}=\frac{p_{3}^{2}}{2 m}+U_{x}+U_{y}=\frac{\left(\mathbf{P}^{\prime}+\mathbf{q}^{\prime}\right)^{2}}{2 m}+U_{x}+U_{y} \\
& E_{4}=\frac{p_{4}^{2}}{2 m}+U_{x}+U_{y}=\frac{\left(\mathbf{P}^{\prime}-\mathbf{q}^{\prime}\right)^{2}}{2 m}+U_{x}+U_{y} .
\end{aligned}
$$

The expression (45) is also the direct transposition of Eq. (26) of 13. After integration and changes of variables similar to the ones given above for the calculation of $S(\eta)$, we reach (20) where

$$
\begin{aligned}
\tilde{S}(\eta)= & \frac{1}{4 \pi^{2} S(\eta)} \frac{e^{\eta}}{\left(1-e^{-\eta}\right)^{2}} \int_{-\sqrt{2 \eta}}^{\sqrt{2 \eta}} d w e^{-w^{2}} \int_{0}^{a} d u(a-u) e^{-u^{2}} \\
& \times\left\{\frac{a+u}{\sqrt{\pi}} e^{-(a-u)^{2}}+\frac{a-u}{\sqrt{\pi}} e^{-(a+u)^{2}}-\left(a^{2}-u^{2}\right)(\operatorname{erfc}(a-u)+\operatorname{erfc}(a+u))\right\} .
\end{aligned}
$$

with $a=\sqrt{2 \eta-w^{2}}$ as in (44). 
FIG. 1. An atomic beam propagates in a transverse magnetic guide. Evaporation eliminates particles whose transverse coordinate exceeds some adjustable value $\Lambda_{\text {evap }}(z)$. The emerging beam is colder and it has a larger phase-space density than the input beam.

FIG. 2. Variations with $\eta$ of the dimensionless parameters $S$ multiplied by 10 for clarity (full line) and $\tilde{S}$ (dashed line). The analytical expressions for these parameters are derived in the appendix.

FIG. 3. Longitudinal velocity as a function of the position $z$ measured in units of the initial mean free path $d_{0}=\sqrt{\pi} /\left(2 n_{0} \sigma\right)$. The unit for velocity is the initial velocity spread $\Delta v_{0}$. The continuous line corresponds to the numerical solution of the $1 \mathrm{D}$ hydrodynamic equation for $\eta=5$. The markers indicate the results of the Monte-Carlo simulation.

FIG. 4. Flux as a function of the position $z$ measured in units of the initial mean free path $d_{0}=\sqrt{\pi} /\left(2 n_{0} \sigma\right)$. The unit of flux is the initial value at $z=0$. The continuous line corresponds to the numerical solution of the $1 \mathrm{D}$ hydrodynamic equation for $\eta=5$. The markers indicate the results of the Monte-Carlo simulation.

FIG. 5. Gain in phase-space density as a function of the position $z$ measured in units of the initial mean free path $d_{0}=\sqrt{\pi} /\left(2 n_{0} \sigma\right)$. The continuous line corresponds to the numerical solution of the $1 \mathrm{D}$ hydrodynamic equation with $\eta=5$. The markers indicate the results of the Monte-Carlo simulation.

FIG. 6. Gain in phase space density as a function of the position $z$ expressed in units of the initial mean free path $d_{0}=\sqrt{\pi} /\left(2 n_{0} \sigma\right)$. These curves are the numerical solutions of Eqs. 26-28) in the case where $\eta$ is fixed.

FIG. 7. For an ideal Bose gas fraction of atoms in the transverse ground state, as a function of the linear density. This figure has been obtained for $k_{B} T=20 \hbar \Omega_{\perp}$, so that $\rho_{\text {lin }}^{(c)} \simeq 536 \lambda^{-1}$. 
[1] For a review, see W. Ketterle and N. Van Druten, Advances in Atomic, Molecular, and Optical Physics, 37, 181 (1996).

[2] M. H. Anderson, J. Ensher, M. Matthews, C. Wieman, and E. Cornell, Science 269, 198 (1995).

[3] C. C. Bradley, C. A. Sackett, and R. G. Hulet, Phys. Rev. Lett. 78, 985 (1997); see also C. C. Bradley et al., Phys. Rev. Lett. 75, 1687 (1995).

[4] K. B. Davis, M.O. Mewes, N. Van Druten, D. Durfee, D. Kurn, and W. Ketterle, Phys. Rev. Lett. 75, 3969 (1995).

[5] D. Fried, T. Killian, L. Willmann, D. Landhuis, S. Moss, D. Kleppner, and T. Greytak, Phys. Rev. Lett. 81, 3811 (1998).

[6] For proposals for atom lasers based either on laser cooling, molecular dissociation, or collisions, see: R. J. C. Spreeuw, T. Pfau, U. Janicke, and M. Wilkens, Euro. Phys. Lett. 32, 469 (1995); M. Olshanii, Y. Castin, and J. Dalibard, in Proceedings of the XII Conference on Laser Spectroscopy, edited by M. Inguscio, M. Allegrini, and A. Sasso (World Scientific, Singapore, 1996); H. M. Wiseman and M. J. Collett, Phys. Lett. A 202, 246 (1995); C. Bordé, Phys. Lett. A 204, 217 (1995); J. I. Cirac and M. Lewenstein, Phys. Rev. A 53, 2466 (1996); M. Holland, K. Burnett, C. Gardiner, J. I. Cirac, and P. Zoller, Phys. Rev. A 54, R1757 (1996); H. M. Wiseman, Phys. Rev. A 56, 2068 (1997); B. Kneer, T. Wong, K. Vogel, W. P Schleich, and D. F. Walls, Phys. Rev. A 58, 4841 (1998).

[7] For experiments on pulsed or quasi-continuous coherent atomic sources extracted from a 3D Bose-Einstein condensate, see: M.-O. Mewes, M. R. Andrews, D. M. Kurn, D. S. Durfee, C. G. Townsend, and W. Ketterle, Phys. Rev. Lett. 78 , 582 (1997); B. P. Anderson and M. Kasevich, Science 282, 1686 (1998); E. W. Hagley, L. Deng, M. Kozuma, J. Wen, K. Helmerson, S. L. Rolston, W. D. Phillips, Science 283, 1706 (1999); I. Bloch, T. W. Hänsch, and T. Esslinger, Phys. Rev. Lett. 82, 3008 (1999).

[8] For a review, see K. Helmerson, D. Hutchinson, K. Burnett, and W.D. Phillips, Physics World, August 1999, p. 31.

[9] This large flux has already been achieved experimentally with continuous sources of slow atoms. See e.g. Z. T. Lu, K. L. Corwin, M. J. Renn, M. H. Anderson, E. A. Cornell, and C. E. Wieman, Phys. Rev. Lett. 77, 331 (1996), and K. Dieckmann, R. J. C. Spreeuw, M. Weidemüller, and J. T. M. Walraven, Phys. Rev. A 58, 3891 (1998).

[10] To compensate for the deceleration of the guided atoms caused by the variation of $B_{0}$ with $z$, one can tilt slightly the guide with respect to the horizontal position. Alternatively the corresponding extra variation of $\bar{p}$ can be incorporated in our treatment.

[11] O. Luiten, M. Reynolds, and J. Walraven, Phys. Rev. A 53, 381 (1996).

[12] Quasi-ergodicity in the 2D evaporation scheme could be obtained by introducing a slight non-axi-symmetric potential, e.g. with a far detuned laser beam. A relative oscillation frequency difference between $x$ and $y$ axis $\Delta \Omega / \Omega_{\perp} \sim \pi \gamma_{\text {coll }} / \Omega_{\perp} \sim 6 \%$ is sufficient to mix the $x$ and $y$ motions between two collisions. If this is achieved a formulation of $2 \mathrm{D}$ evaporation in terms of the transverse energy $\epsilon_{\perp}$ is possible. Note however that the calculations are in this case much more involved than for $1 \mathrm{D}$ or $3 \mathrm{D}$ evaporation.

[13] P. Pinkse, A. Mosk, M. Weidemüller, M. Reynolds, T. Hijmans, and J. Walraven, Phys. Rev. A 57, 4747 (1998).

[14] The approximate exponential variations found in section $\mathrm{V}$ allow an estimation of the loss rate $\Gamma_{\mathrm{sp}}$ of particles due to spilling. One finds $\Gamma_{\mathrm{sp}} / \Gamma \simeq 2 \eta(\eta+\tilde{S}-1) /\left(5 e^{\eta}-5\right)$ which is on the order of $6 \%$ for $\eta=5$.

[15] G. A. Bird, Molecular Dynamics and the Direct Simulation of Gas Flows (Clarendon Press, Oxford, 1994).

[16] H. Wu and C.J. Foot, J. Phys. B: At. Mol. Opt. Phys. 29, L321 (1996).

[17] H. Wu, E. Arimondo and C.J. Foot, Phys. Rev. A 56, 560 (1997).

[18] D. Guéry-Odelin, J. Söding, P. Desbiolles and J. Dalibard, Optics Express 2, 323 (1998).

[19] K. Huang, Statistical Mechanics, John Wiley (New York, 1963).

[20] This should not be confused with the 3D thermodynamic limit for this problem, which is obtained by letting $N, L \rightarrow \infty, \Omega_{\perp} \rightarrow 0$, while keeping a constant on-axis density and a constant product $L \Omega_{\perp}$.

[21] W. Ketterle and N. J. Van Druten, Phys. Rev. A 54, 656 (1996). 


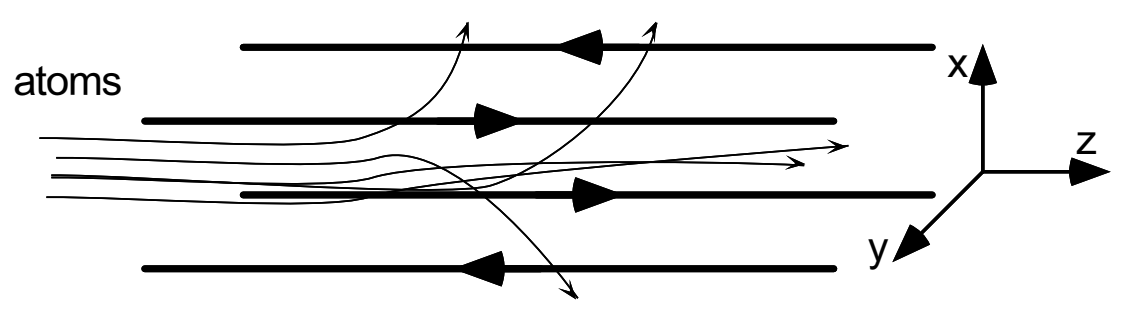




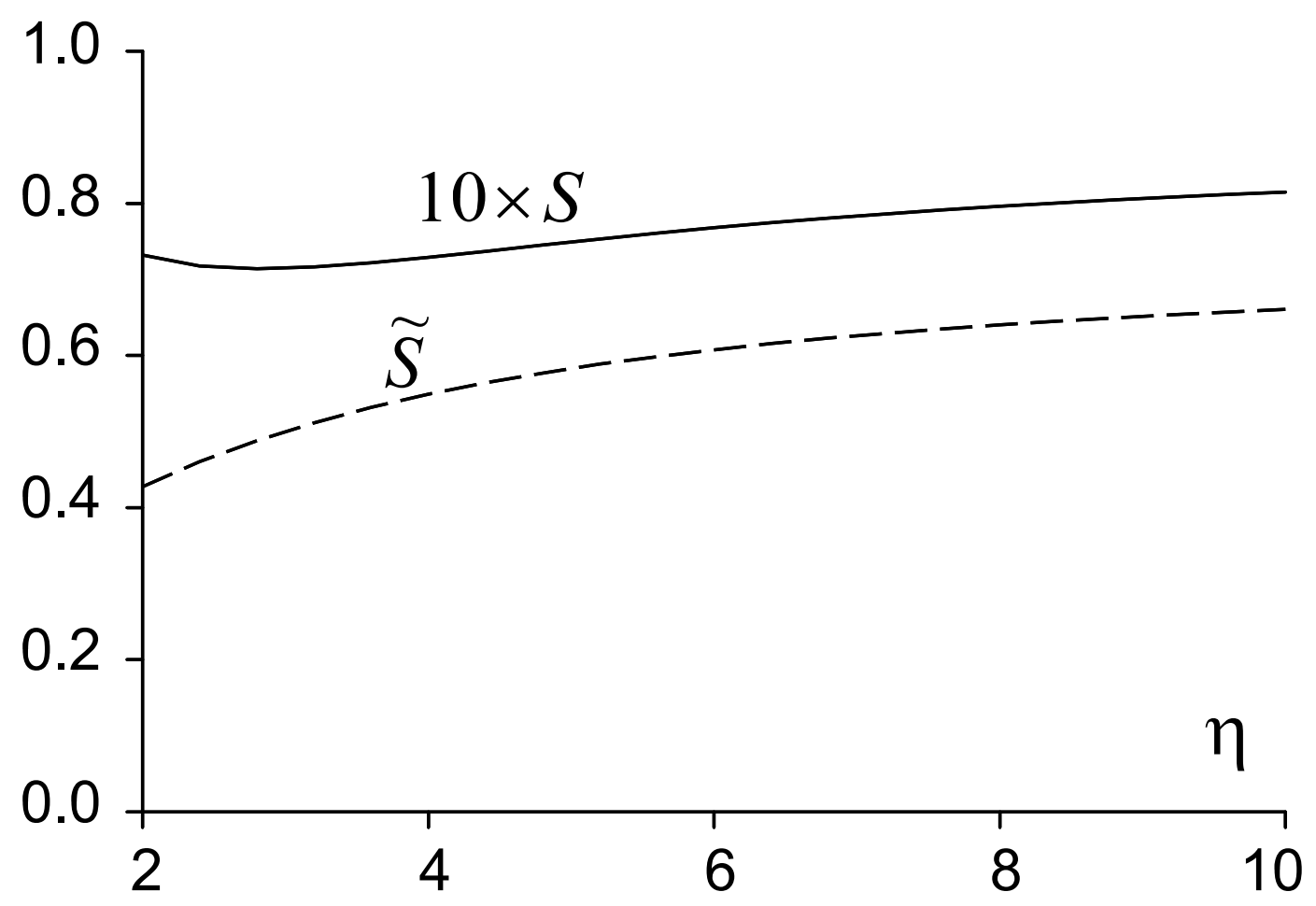




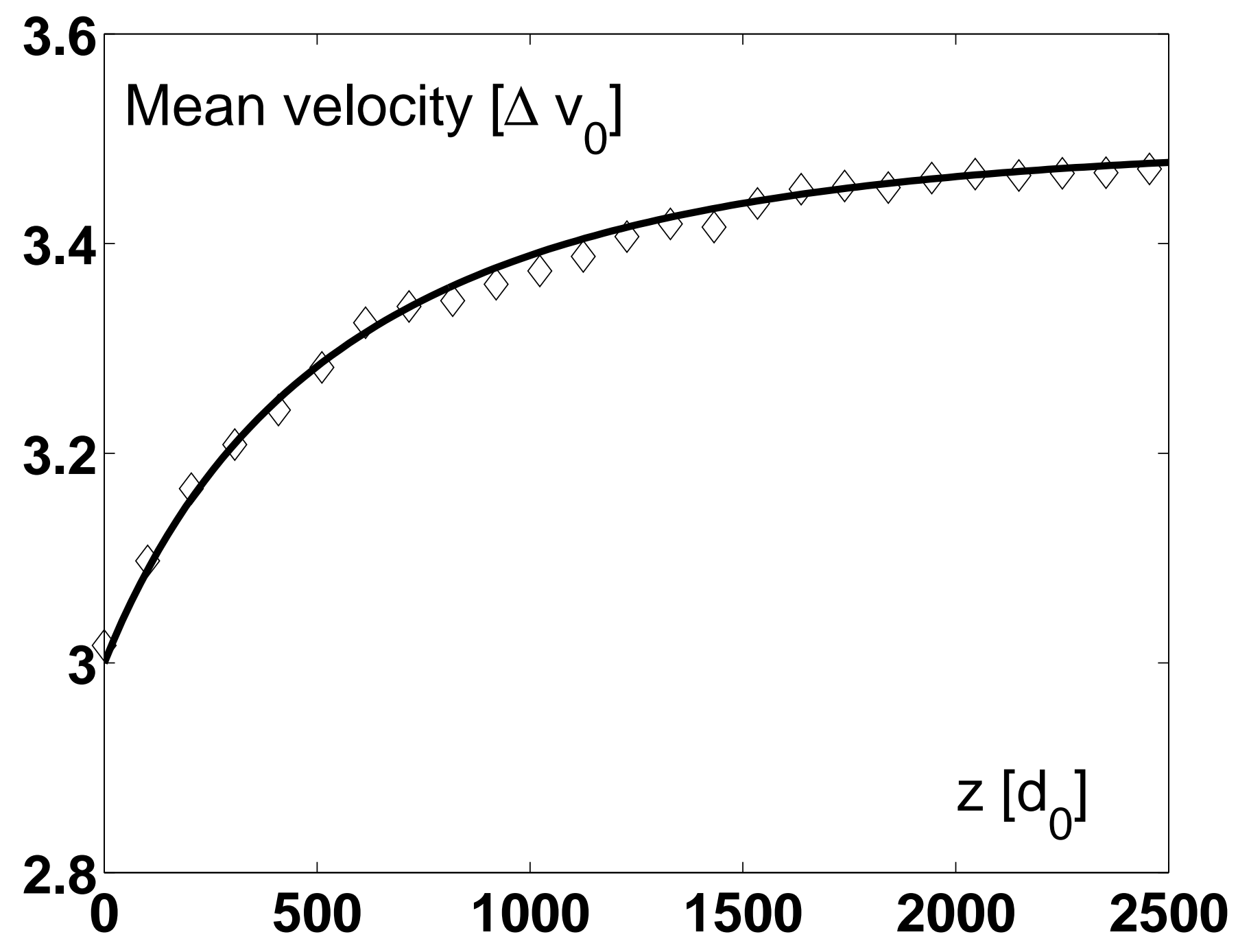




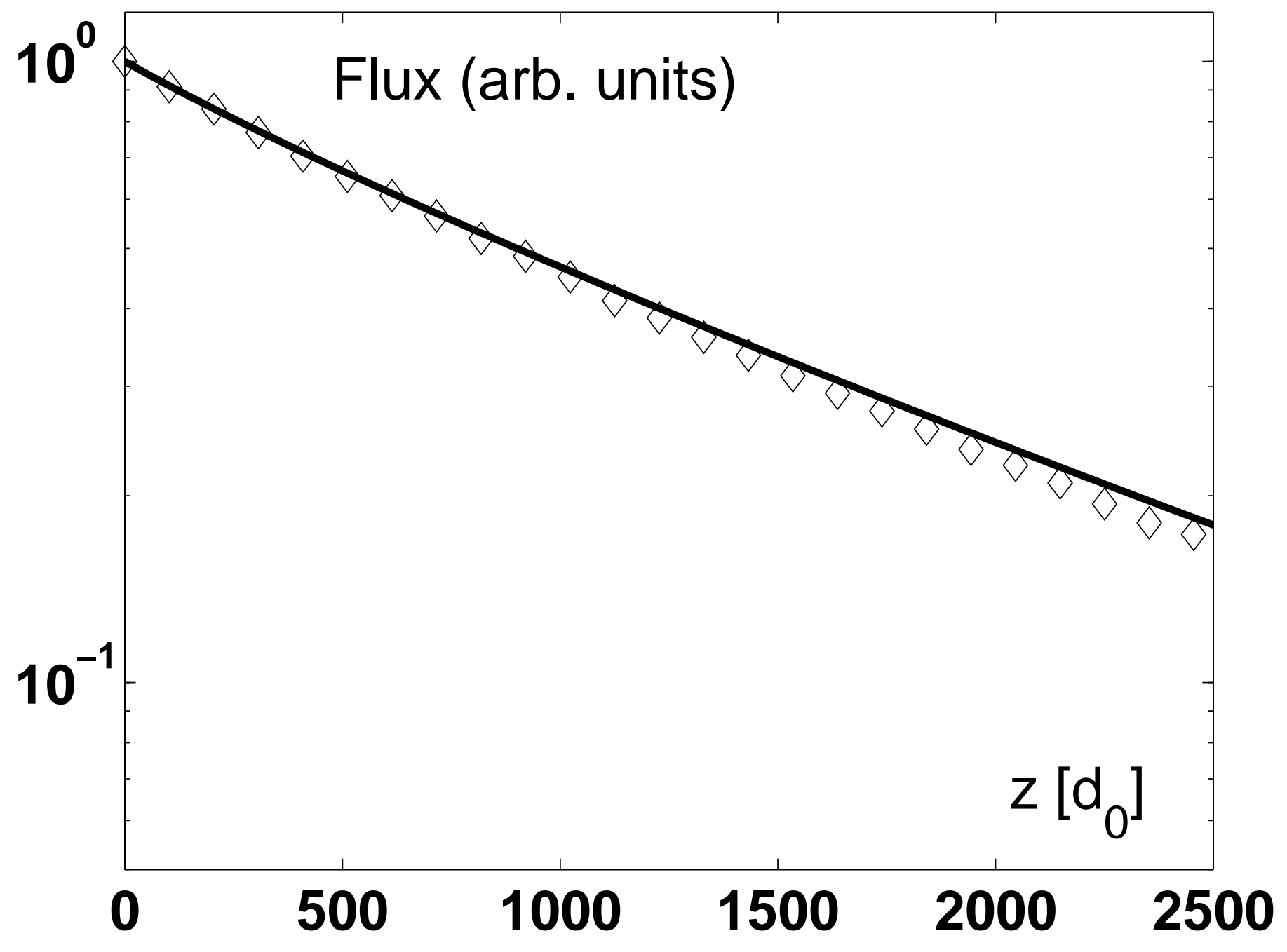




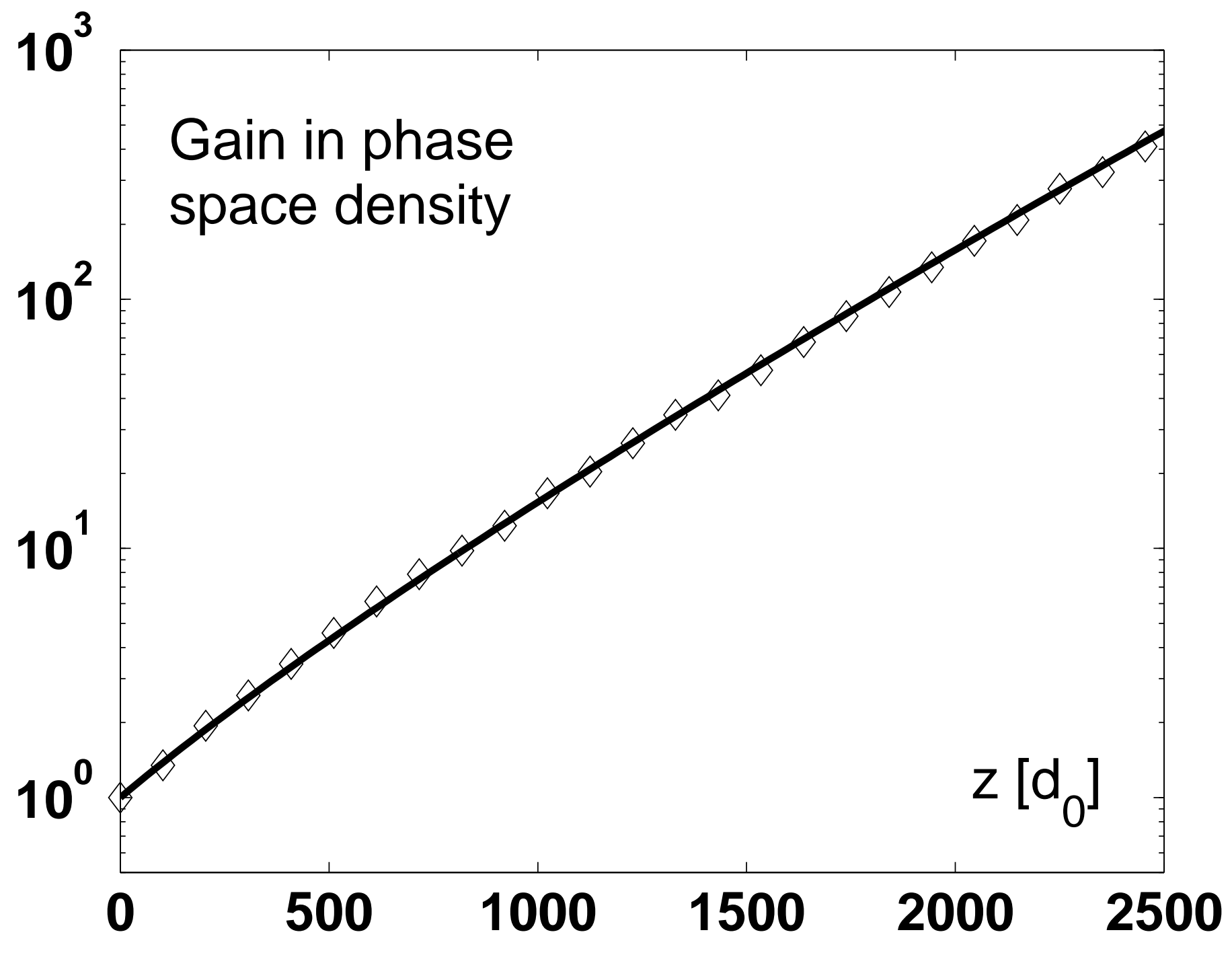




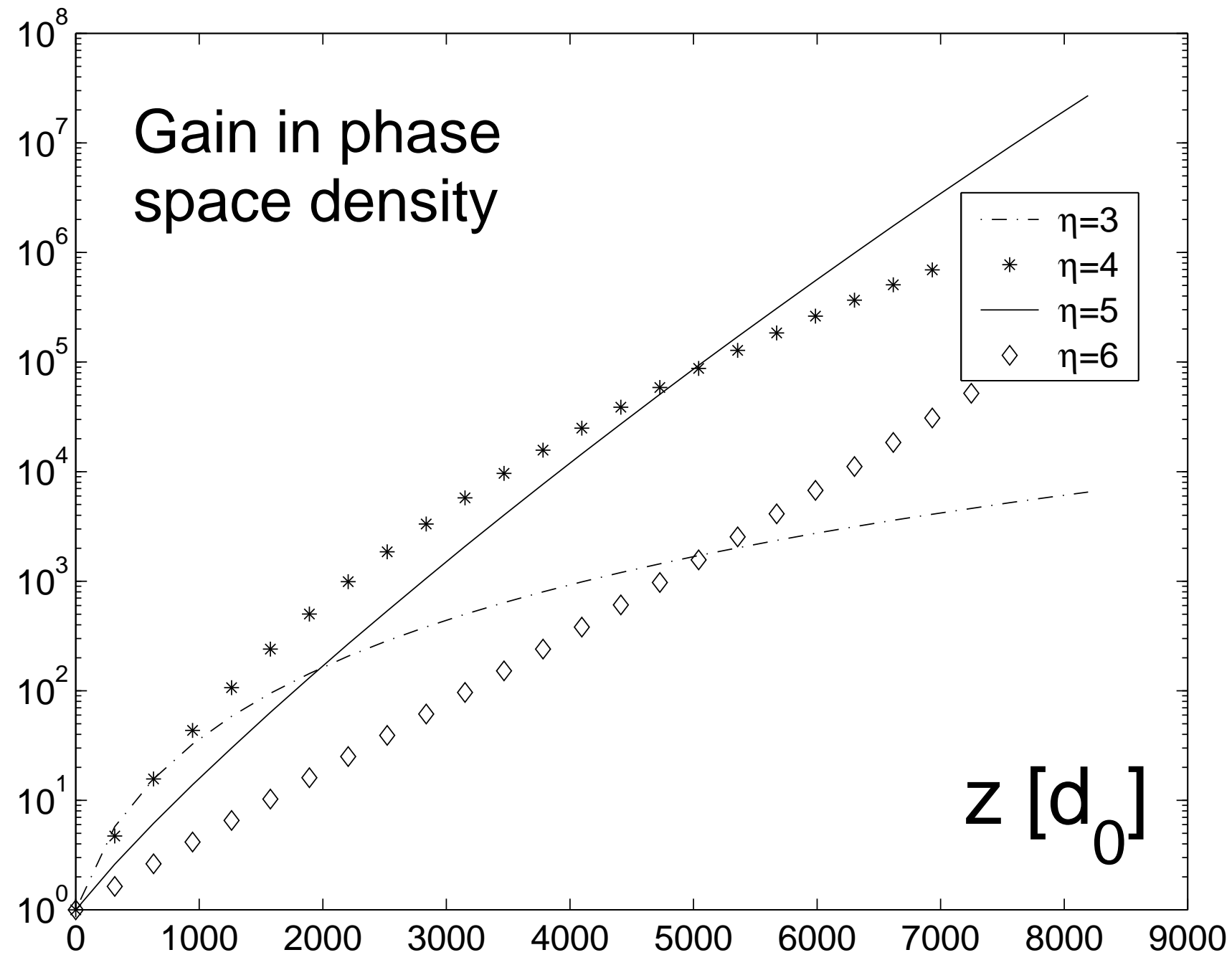




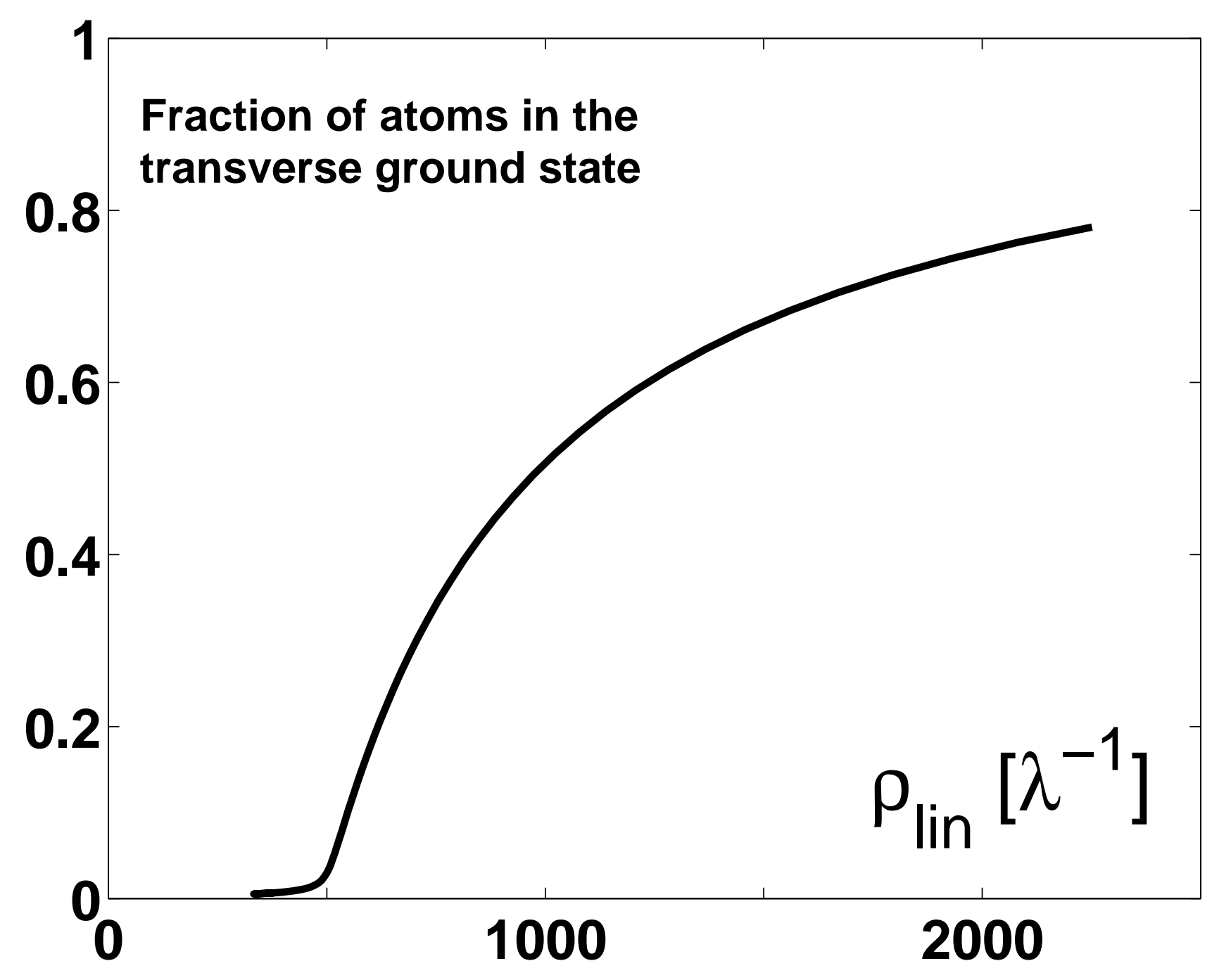

